\title{
Dos celebraciones: Décimo aniversario de AIEM y nueva Editora
}

\author{
Núria Planas, Universitat Autònoma de Barcelona
}

\section{Dos celebraciones: Décimo aniversario de AIEM y nueva Editora}

\section{Resumen}

En este Editorial, reflexiono sobre diversas experiencias de aprendizaje surgidas del período de cuatro años como Editora de Avances de Investigación en Educación Matemática. En particular, doy gracias a los autores, revisores y editores asociados, y a todos aquellos que leen y consideran los manuscritos que se publican en la revista. AIEM está a punto de alcanzar sus 10 años de vida y este es un motivo de celebración, junto con la entrada de la nueva Editora, Ceneida Fernández. Tras superar con éxito muchos retos y formular muchos otros, AIEM ya no es una 'niña pequeña' en la comunidad internacional de revistas de investigación en educación matemática.

Palabras clave. Revista científica; esencia del trabajo de edición; retos de equipo; comunidad de investigación en educación matemática; 10 años de AIEM.

\section{Two celebrations: Ten-year anniversary of AIEM and new Editor-in-Chief}

\section{Abstract}

In this Editorial, I reflect on diverse learning experiences gained throughout the 4-year term as Editor-in-Chief of Advances of Research in Mathematics Education. I particularly acknowledge the authors, the reviewers and the associate editors, and all those who read and consider the manuscripts published in the journal. AIEM has managed to continue publishing for 10 years and this is a reason to celebrate, as well as the formal appointment of the new Editor-in-Chief, Ceneida Fernández. Many challenges have successfully been addressed and formulated, which make AIEM not a 'kid' anymore in the international community of mathematics education research journals.

Keywords. Scientific journal; core of editing work; team challenges; community of mathematics education research; 10 years of AIEM.

\section{Gracias por este leyendo este Editorial}

William Faulkner explicaba que escribía sin descanso cuando tenía algo importante que contar que supusiera un giro o una precisión en el argumento de sus poemas e historias (Blotner, 1991/2005). Pues bien, la Editora y antes el Editor de AIEM hemos trabajado sin descanso a la vez que hemos escrito pocos Editoriales. Los números de AIEM no suelen tener Editorial excepto en ocasiones singulares donde aspectos de política editorial o giros de algún tipo se consideran lo bastante importantes para ser contados en primera persona. El cambio de Editora en enero de 2022 es una de estas ocasiones singulares.

Hace poco más de cuatro años, se producía el primer relevo tras los años fundacionales y de liderazgo editorial de Lorenzo Blanco, compañero de la Universidad de Extremadura. Producía algo de vértigo tomar las riendas de un proyecto colectivo, compartido e intergeneracional, que era el de todas las compañeras y compañeros de la Sociedad Española de Investigación en Educación Matemática. Lorenzo ha seguido siempre estando para lo que se necesitara y, en gran medida, gracias a un relevo ordenado y acompañado, los logros se han ido sucediendo a modo de cúmulo de razones sobre por qué era adecuada una revista científica más de educación matemática. Hemos conseguido, por ejemplo, la entrada en la base ERIH y la evaluación positiva de la 
base Scopus con cambio reciente a tercer cuartil; con esto, los indicadores bibliométricos nos empiezan a ser favorables a la vez que disponemos de herramientas sofisticadas de seguimiento de la revista y de sus artículos. Así llegamos a la antesala de la celebración del décimo aniversario de AIEM y al turno de nuestra compañera Ceneida Fernández, de la Universitat d'Alacant. Ceneida cuenta desde ya con el apoyo de los dos Editores previos de AIEM, del actual Equipo Editorial y de la Sociedad Española de Investigación en Educación Matemática.

Aprovecho aquí para agradecer su labor y talante a los compañeros presentes y pasados del Equipo Editorial. Hay, sin embargo, muchas más sillas en la mesa. El servicio de los revisores para con la revista y el área es fundamental. Como escribí en un Editorial anterior (Planas, 2019), el tiempo y trabajo dedicados a los autores y a la mejora de sus manuscritos son un puntal de AIEM, a menudo un puntal anónimo y siempre altruista en estos tiempos en los que todos estamos increíblemente ocupados. Es justo reconocer que la calidad de nuestra revista es resultado de la calidad de las investigaciones comunicadas en los artículos que se publican, pero también de las minuciosas rondas de revisión y de discusión con los autores, sus ideas y sus modos de ponerlas por escrito para que se entiendan correctamente o tal como ellos las piensan. El proceso involucrado en "la investigación hecha artículo" no es sencillo, ni es más importante que la propia investigación y su efecto en quienes participan, ni tampoco es más complejo que los retos de "la charla plenaria hecha artículo" o de "la comunicación en congreso hecha artículo".

Dicho lo anterior y siendo básicas todas las sillas de la mesa en el proceso de revisión, la decisión final sobre la publicación de cada artículo en AIEM recae en el Editor. Esta responsabilidad obliga a leer los artículos uno por uno en sus versiones 'finales provisionales', los informes de revisión, las cartas de los editores asociados y las cartas con relatorios de cambios de los autores. Además de colaborar con autores, revisores y editores asociados, otra responsabilidad durante estos años, que no supe anticipar, ha sido la de responder a los investigadores que envían correos extensos donde explican el tipo de estudio que han realizado o que están desarrollando, o bien que envían directamente el texto de una comunicación en congreso, y preguntan si un artículo acerca de ese estudio o con base en esa comunicación podría resultar apropiado para la revista AIEM. He tratado con sumo cuidado cada uno de estos correos y he entrado en largas conversaciones con estos investigadores, con la idea de que algún día acabaran enviando un artículo a AIEM que pudiera entrar en el procedimiento de revisión. Unas veces esto ha comportado animar a que precisaran qué partes o ideas concretas de su investigación tenían intención de publicar y a que argumentaran por qué consideraban que esas partes o ideas eran científicamente novedosas para la comunidad del área y merecían publicarse. Estas son preguntas que yo misma me hago cada vez que me planteo preparar un artículo y que no son triviales ni éticamente neutras.

Lejos de esquivar la responsabilidad de lo que podría haberse hecho mejor o de cuánto más podría haberse hecho en estos cuatro años, de verdad pienso que la Editora ocupa una silla de las muchas que hay en la mesa. De un modo u otro, todos (autores, editores asociados, revisores, lectores...) somos Editores de AIEM. Ayuda bastante estar acostumbrados a editar textos que generamos dentro de otros registros como madres, como amigos, como vecinos... Al fin y al cabo, editar es el proceso de simplificar y de hacer más comprensible un texto dentro de un registro específico y en un contexto de cultura particular. Desde 'El libro de las tierras vírgenes' hasta 'El libro de la selva", por ejemplo, Rudyard Kipling editó varias veces el título en inglés hasta encontrar un registro adecuado para referirse a las aventuras de Mowgli; y volvió a editar 
páginas del libro en sus últimos años para pedir que se borraran las esvásticas que él tanto dibujaba por ser símbolo de buena suerte y de bienestar en su India natal (Kipling, 1937/2020). Editamos continuamente en contextos particulares porque editar está en la naturaleza de comunicar. Es posible que muchas de las veces en las que hablamos con nuestros vecinos sobre un tema importante y no nos sentimos satisfechos acerca de cómo se nos está entendiendo, haya faltado por nuestra parte editar un poco más lo que queríamos comunicar, o tal vez haber editado con algo de retroacción.

En los textos para una revista científica, la retroacción está garantizada en las distintas fases de producción. Esta retroacción debe estar situada en el conocimiento del estado del arte. No por evidente este criterio científico pierde contundencia ni debemos dejar de insistir en él, sobre todo porque al estar increíblemente ocupados podríamos relajarnos. Recuerdo que el primer artículo que preparé iba con un título de casi tres líneas que, tras un seminario con otros doctorandos, comprobé que no gustaba ni se entendía como yo esperaba. El artículo, que no llegó a publicarse, partía del argumento de que no había evidencias acerca de un cierto fenómeno del aula de matemáticas. En los dos informes de expertos que recibí y en la carta del Editor Asociado, este argumento quedó amablemente refutado y empecé con humildad a estudiar las referencias de trabajos que los dos revisores anónimos habían coincidido en sugerir. Todos necesitamos Editores y durante toda nuestra trayectoria investigadora, a veces, para lograr algo aparentemente sencillo como redactar un título que sea preciso o incluso que no aleje a los lectores a los que va dirigido el texto. Y, aun así, editar dentro del registro específico de la investigación en educación matemática es mucho más que un mero ejercicio académico o lingüístico; se requiere conocer la singularidad del área y estar al corriente de y estudiar los avances en nuestro dominio específico fuera de los realizados exclusivamente por nosotros o por el grupo con el que colaboramos. No se puede hacer investigación en educación matemática ni escribirla en forma de artículo sin recurrir a la investigación y a los artículos de los otros expertos. Los otros expertos son nuestro gran recurso, nuestra posibilidad de que se genere movimiento vygotskiano en nuestra Zona de Desarrollo Próximo (Wertsch, 1985), de que aprendamos.

Deseo que, cada vez más y con la ayuda de todos, AIEM devenga una de nuestras revistas científicas de referencia, tanto para publicar nuestras investigaciones como para leer y citar artículos aparecidos en la revista que sean relevantes en nuestras respectivas líneas de estudio. No sé muy bien cuán lejos o cerca estamos de cumplir las expectativas y promesas que llevaron a aventurar en 2012 que una revista científica más de educación matemática era adecuada; esa es una valoración que podemos realizar cada uno de nosotros. Los artículos son de libre acceso e incluso puede resultar relativamente fácil acercarse a sus autores y a participantes del Equipo Editorial en alguno de los Simposios de la Sociedad Española de Investigación en Educación Matemática. Como ocurre en estos Simposios, la región iberoamericana es la más representada a nivel de autores, revisores y lectores; este es claramente un rasgo distintivo de AIEM y de los entornos en los cuales se piensan y desarrollan la mayoría de investigaciones que se publican. A lo largo de veinte números y diez años, bastantes artículos de AIEM nos enseñan cómo es, cómo se transforma y cómo se preserva la investigación en educación matemática en esta parte del mundo. No por ello, sin embargo, AIEM aspira menos a ser una voz plural de referencia en la comunidad internacional, especialmente a través de los monográficos con periodicidad anual.

Me queda desear dos últimas cosas: que Ceneida Fernández tenga una formidable experiencia en la etapa de Editora de AIEM que pronto iniciará, y que todos sigamos siendo una comunidad de Editores en la que nuestra compañera pueda apoyarse. De- 
bemos editar nuestros artículos y los de los otros expertos, con generosidad de tiempo y criterio científico. Así, AIEM contribuirá a que surjan más y nuevas preguntas de investigación, a la vez que no dejará de respaldar el estudio de preguntas básicas de la educación matemática que siguen abiertas. Involucrarnos en la apertura de espacios académicos a nuevas preguntas tal vez sirva para que seamos también un foro de la investigación en educación matemática que fundamenta prácticas y textos responsables con las crisis ecológica y climática y con las geografías de exclusión cultural y social.

Sin más, os doy las gracias por haber leído este Editorial y por apoyar a AIEM.

\section{Agradecimientos}

A Nuria Climent y a Ceneida Fernández, por aceptar ser las otras expertas en sus comentarios a una versión primera de este Editorial.

\section{Referencias}

Blotner, J. (2005). Faulkner: A biography. University Press of Mississippi. (Versión original 1991).

Kipling, R. (2020). Something of myself. For my friends, known and unknown. The complete unfinished autobiography [ed. C. Starr]. Pantianos Classics. (Versión original 1937)

Planas, N. (2019). Avances de Investigación en Educación Matemática: Tres transiciones cualitativas en proceso. AIEM, 16, 1-4.

https://doi.org/10.35763/aiem.v0i16.285

Wertsch, J. V. (1985). Vygotsky and the social formation of mind. Harvard University Press.

\section{Referencia de la autora}

Núria Planas, Universitat Autònoma de Barcelona (España). nuria.planas@uab.cat 